EPJ Web of Conferences 49, 16009 (2013)

DOI: $10.1051 /$ epjconf/20134916009

(c) Owned by the authors, published by EDP Sciences, 2013

\title{
Prompt Photon Production at the Tevatron and LHC
}

\author{
Ashish Kumar (for the ATLAS, CDF and D0 collaborations) \\ ${ }^{1}$ Department of Physics, The State University of New York at Buffalo, Buffalo, NY 14260, USA
}

\begin{abstract}
Prompt photon production has been studied by the CDF and $\mathrm{D} \emptyset$ experiments at the Fermilab Tevatron collider in $p \bar{p}$ collisions at the centre of mass energy of $\sqrt{s}=1.96 \mathrm{TeV}$ and by the ATLAS experiment at LHC in $p p$ collisions at $\sqrt{s}=7 \mathrm{TeV}$. Measurements of the inclusive photon, inclusive photon plus jet, photon plus heavy flavor jet, and diphoton production cross sections are discussed. The results are compared to the perturbative QCD calculations and predictions from Monte Carlo generators.
\end{abstract}

\section{Introduction}

Prompt photons [1] are defined as photons produced in the beam particle collisions and not originating from particle decays. They include both direct photons, which originate from the hard interaction, and fragmentation photons, which arise from the parton fragmentation. However, application of photon isolation requirements substantially reduce the contribution of fragmentation photons. At the lowest order (LO) in pQCD, such events are produced primarily through the Compton scattering $g q \rightarrow q \gamma$. In $p \bar{p}$ collisions at the Tevatron, annihilation contribution $q \bar{q} \rightarrow g \gamma$ dominates at high photon transverse momentum $\left(p_{T}^{\gamma}\right)$. Study of prompt photons, therefore, provide precision tests of our theoretical understanding based on pQCD as well as possible constraint on the relatively poorely measured gluon density of the proton since gluon is involved at tree level in contrast to deep inelastic scattering (DIS) and Drell-Yan (DY) processes where gluon is involved only at a higher order.

Photons in final state may be an important sign of new particles and /or physics beyond the Standard Model. Understanding the QCD production mechanisms of photons is, therefore, a prerequisite to searches for new physics. Prompt photon production cross section measurement is extremely challenging due to enormous background from jets fragmenting into a leading $\pi^{0}$ or $\eta$, particularly at low $p_{T}$. Photons arising from the decays of $\pi^{0}$ and $\eta$ are largely suppressed by the photon selection requirements applied to data, and especially by the photon isolation, since these mesons are produced mainly within jets during fragmentation and are surrounded by other particles. Even after very tight selection criteria, highly electromagnetic jets provide a formidable background due to their large cross section. The two showers from the energetic $\pi^{0}$ or $\eta$ decaying to two photons coalesce in the calorimeter and mimic single photon shower. Consequently, they can not be rejected on an event-by-event basis and we must perform a statistical background subtraction in order to measure the cross section.

\section{Inclusive photon production cross section}

The ATLAS experiment has performed the preliminary measurement of the inclusive isolated photon cross section in $p p$ collisions at $\sqrt{s}=7 \mathrm{TeV}$ using $35 \mathrm{pb}^{-1}$ of data collected during 2010 [2]. The measurement covers the pseudorapidity $(\eta)$ ranges $\left|\eta^{\gamma}\right|<1.37$ and $1.52<\left|\eta^{\gamma}\right|<2.37$ in the transverse energy range $45 \leq E_{T}^{\gamma}<400 \mathrm{GeV}$. This supersedes the previous published measurement [3] using $0.88 \mathrm{pb}^{-1}$ of data which covered the kinematic region $15 \leq E_{T}^{\gamma}<100 \mathrm{GeV}$ in three $\eta^{\gamma}$ bins: $\left|\eta^{\gamma}\right|<0.6$, $0.6 \leq\left|\eta^{\gamma}\right|<1.37$ and $1.52 \leq\left|\eta^{\gamma}\right|<1.81$. Both the measurements are found to be consistent within uncertainties in the overlapping region. In both analyses, the JETPHOX Monte Carlo program [4] is used for the NLO predictions of cross section with different sets of parton distribution functions (PDFs). The predictions with CTEQ6.6 PDFs agree in all cases with the observed cross sections for $E_{T}^{\gamma}>25 \mathrm{GeV}$, while for $E_{T}^{\gamma}<25 \mathrm{GeV}$, the predicted cross sections are higher than the measured ones. Similar results have been obtained with MSTW2008 and NNPDF 2.0 PDFs. The theoretical uncertainty is dominated by the scale uncertainty $(10 \%)$. From the experiemental side, the statistical uncertainty is negligible, the systematic uncertainty being dominated by the photon purity and photon efficiency estimations.

\section{Photon plus jet production cross section}

The ATLAS experiment has measured the production cross section of an isolated photon in association with jets in $\mathrm{pp}$ collisions at $\sqrt{\mathrm{s}}=7 \mathrm{TeV}$ based on $37 \mathrm{pb}^{-1}$ of data [5]. Photons are reconstructed in the $\eta^{\gamma}$ range 
$\left|\eta^{\gamma}\right|<1.37$ with $E_{T}^{\gamma}>25 \mathrm{GeV}$. Jets are reconstructed in the rapidity range $\left|y^{j e t}\right|<4.4$ with transverse momentum $p_{T}^{\text {jet }}>20 \mathrm{GeV}$. A minimum separation of $\Delta R>1.0$ in the $\eta, \phi$ plane is required between the leading jet and the photon. The differential cross section as function of $E_{T}^{\gamma}$ is measured for three different rapidity ranges of the leadingjet: $\left|y^{j e t}\right|<1.2,1.2 \leq\left|y^{j e t}\right|<2.8$, and $2.8 \leq\left|y^{j e t}\right|<4.4$. For each rapidity configuration, the same-sign $\left(\eta^{\gamma} y^{\text {jet }} \geq 0\right)$ and opposite-sign $\left.\eta^{\gamma} y^{\text {jet }}<0\right)$ are studied separately. This subdivision allows the comparison between data and theory predictions in configurations where the relative contribution of the fragmentation component to the cross section and the explored ranges of the incoming parton momentum fraction $\mathrm{x}$ are different. Fig. 1 shows data to theory comparison for the two photon-jet angular configurations. The NLO pQCD cross sections provided by JETPHOX are in fair agreement with the measurements considering the typical (10\% to $30 \%$ ) experimental and theoretical uncertainties. As already observed in previous measurements of inclusive photon production cross section at the LHC, the NLO calculation consistently overestimates the measured
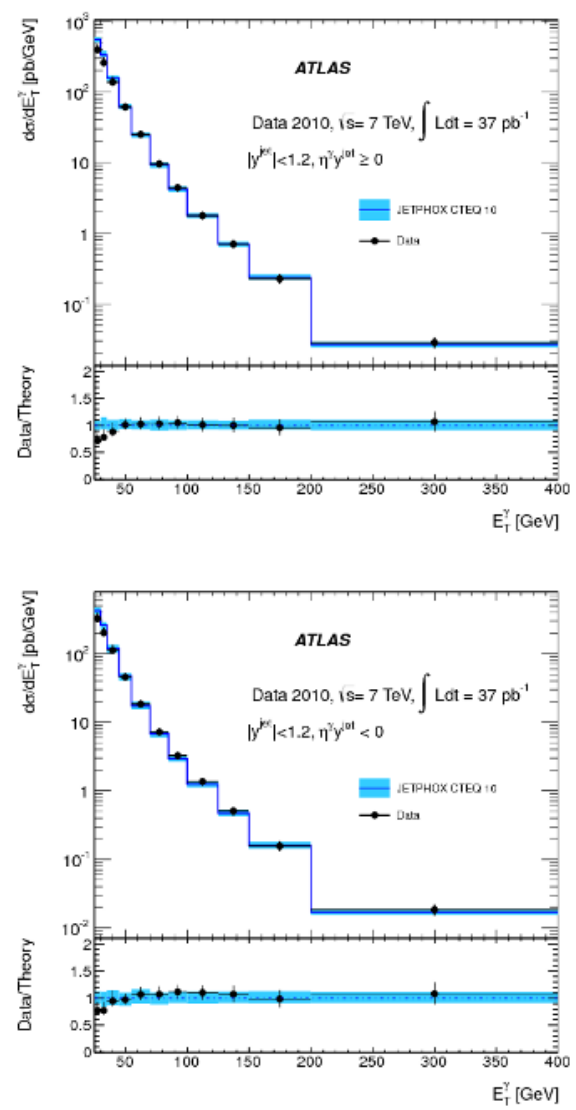

Figure 1. Experimental (black dots) and theoretical (blue line) photon plus jet production cross sections, for $\left|y^{j e t}\right|<1.2$ samesign (Upper) and opposite-sign (Lower) configurations. The black error bars represent the total experimental uncertainty. The blue bands show the total uncertainties on the theoretical predictions obtained with JETPHOX. Bottom graphs: ratio between the measured and predicted cross sections. The blue band shows the theoretical uncertainties while the error bars show the experimental uncertainties on the ratio. cross section in the $E_{T}^{\gamma}<45 \mathrm{GeV}$, possibly suggesting the need for including higher order corrections in the theoretical calculations.

\section{Photon plus heavy flavor jet production cross section}

Study of direct photon production in association with a heavy quark $Q$ ( $b$ or $c$ ) provides information about the $b, c$ and gluon densities within the colliding hadrons [6]. At $p_{T}^{\gamma}<100 \mathrm{GeV}$, they are produced primarily through the Compton scattering process $g Q \rightarrow \gamma Q$, while at high $p_{T}^{\gamma}$, the dominant process is quark-antiquark annihilation $q \bar{q} \rightarrow \gamma g \rightarrow \gamma Q \bar{Q}$. Measurement of $\gamma+Q+X$ production cross section is thus sensitive to the $b, c$ and gluon PDFs and the rate of gluon splitting to heavy quarks, which have substantial uncertainties.
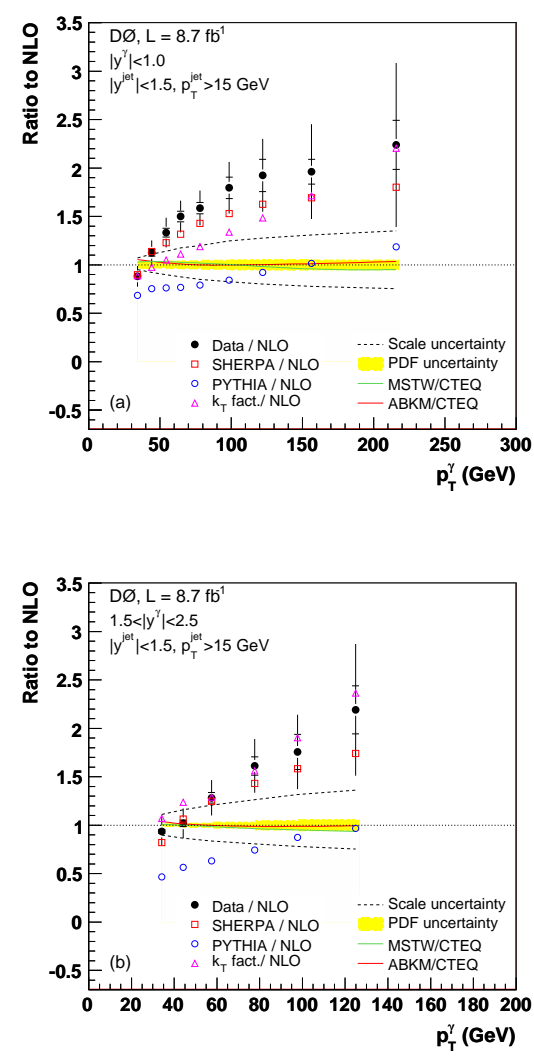

Figure 2. The ratio of $\gamma+b$-jet differential cross sections between data and NLO predictions with uncertainties for the rapidity regions $\left|y^{\gamma}\right|<1.0$ (Upper) and $1.5<\left|y^{\gamma}\right|<2.5$ (Lower). The uncertainties on the data include both statistical (inner error bar) and full uncertainties (entire error bar).

\section{1 $\gamma+$ b-jet cross section measurement}

The D0 experiment has published the measurement of the differential cross section $\mathrm{d} \sigma / \mathrm{d} p_{T}^{\gamma}$ for the inclusive production of an isolated photon in association with a $b$-quark jet as a function of $p_{T}^{\gamma}$ using $8.7 \mathrm{fb}^{-1}$ of data from $p \bar{p}$ collisions at $\sqrt{s}=1.96 \mathrm{TeV}$ [7]. The measurement considers 
central photons with rapidities $\left.\left|y^{\gamma}\right|<1.0\right)$ and $30<p_{T}^{\gamma}<$ $300 \mathrm{GeV}$ as well as forward photons with $1.5<\left|y^{\gamma}\right|<2.5$ and $30<p_{T}^{\gamma}<200 \mathrm{GeV}$. The $b$-quark jets are required to have $p_{T}>15 \mathrm{GeV}$ and rapidity $\left|y^{\text {jet }}\right|<1.5$. The CDF experiment has also performed the preliminary measurement of the $\gamma+b$-jet cross section in the similar kinematic region for only central photons using $9.1 \mathrm{fb}^{-1}$ of collison data [11]. The large data sample and more advanced photon and $b$-jet identification techniques lead to more precise measurements in the much extended kinematic region previously unexplored. In this analysis, there are two main background sources: jets faking photons and light flavor jets faking heavy flavor jets. The analysis adopts two-step procedure for the determination of $b$-jet identification. The $\gamma+$ jet events are required to have at least one jet passing the $b$-tagging selection to enrich the sample with heavy flavor jets. The fraction of $b$-jet is determined from fitting the invariant mass of tracks associated with the secondary vertex $\left(M_{S e c V t x}\right)$ using templates of jet flavors.
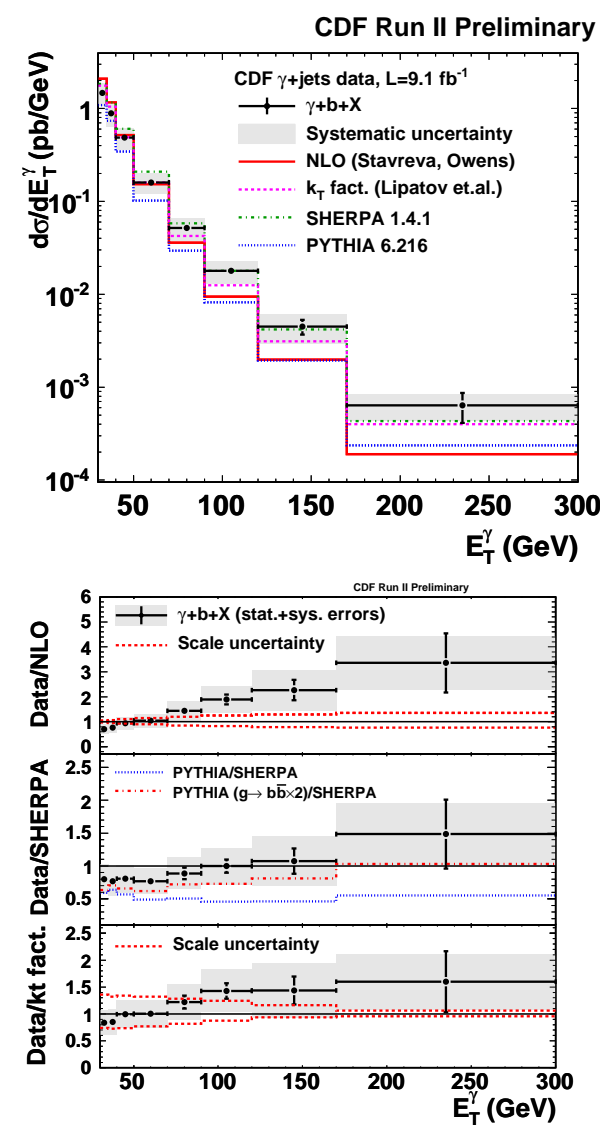

Figure 3. The measured differential cross sections compared with four theoretical predictions. The absolute comparisons are shown in the upper plot and the ratios of data over the theory are shown in the lower plot. The shaded area around the data points indicates total systematic uncertainty of the measurement.

The measured cross sections are compared with the NLO pQCD calculations as well as predictions based on $k_{T}$-factorization approach [9] and those from SHERPA [10] and PYTHIA Monte Carlo event generators. Fig. 2 shows the comparison of the measured cross sections by the D0 with predictions for central and forward photons. Fig. 3 shows similar comparison for the CDF results. As can be seen that the NLO predictions agree with data at low $p_{T}^{\gamma}$ but fail to describe for higher $p_{T}^{\gamma}$. The results indicate a need for higher order perturbative QCD corrections in the large $p_{T}^{\gamma}$ region, that is dominated by the annihilation process $q \bar{q} \rightarrow \gamma g(g \rightarrow b \bar{b})$, and resummation of diagrams with additional gluon radiation. The predictions from the $k_{T}$-factorization approach and Sherpa are in better agreement with data. Fig. 3 also shows that after enhancing the rate of $g \rightarrow b \bar{b}$ splitting to twice, the PYTHIA predictions agree better with data in shape.

\section{$4.2 \gamma+\mathrm{C}$-jet cross section measurement}

Based on the same data set as used in the $\gamma+b$-jet cross section measurement, D0 [8] and CDF [11] have measured the differential cross section $\mathrm{d} \sigma / \mathrm{d} p_{T}^{\gamma}$ for the inclusive production of an isolated photon in association with a $c$-quark jet as a function of $p_{T}^{\gamma}$ using $8.7 \mathrm{fb}^{-1}$ of data. The measurement is performed for photons within rapidities $\left|y^{\gamma}\right|<1.0$ and $30<p_{T}^{\gamma}<300 \mathrm{GeV}$, while the $c$-jet is required to have $\left|y^{\mathrm{jet}}\right|<1.5$ and $p_{T}^{\text {jet }}>15 \mathrm{GeV}$.
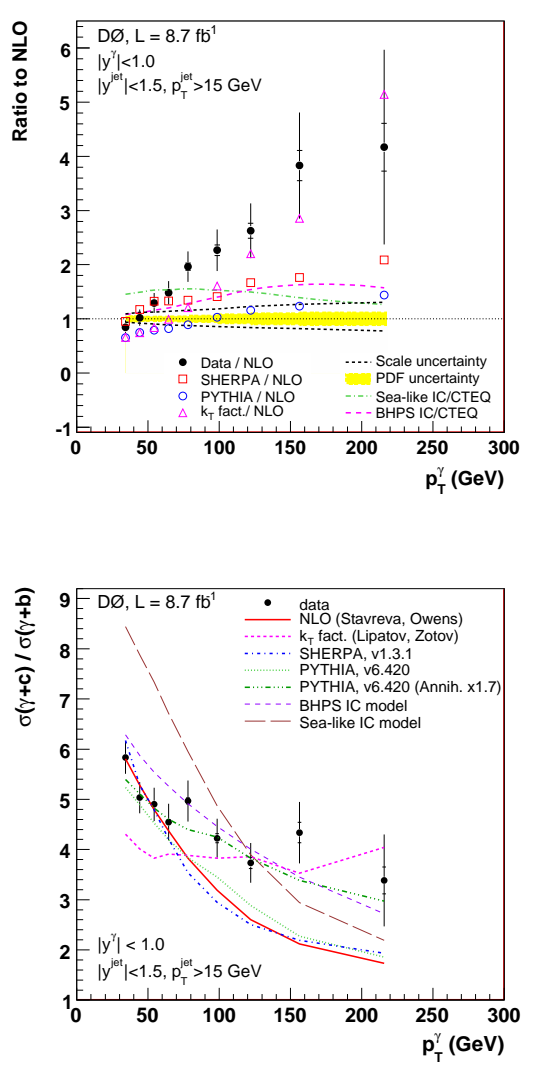

Figure 4. The ratio of $\gamma+c$-jet differential cross sections between data and NLO predictions with uncertainties for the rapidity regions $\left|y^{\gamma}\right|<1.0$ (Upper). The uncertainties on the data include both statistical (inner error bar) and full uncertainties (entire error bar). The ratio of differential cross sections for $\gamma+c$-jet to $\gamma+b$-jet production (Lower).

The measured cross sections are in agreement with the NLO predictions within theoretical and experimental 
uncertainties in the region of $30<p_{T}^{\gamma} \lesssim 70 \mathrm{GeV}$, but show systematic disagreement for larger $p_{T}^{\gamma}$ (see Fig.4 and Fig.5). The results suggest a need for higher-order perturbative QCD corrections in the large $p_{T}^{\gamma}$ region, which is dominated by the annihilation process $q \bar{q} \rightarrow \gamma g$ (with $g \rightarrow c \bar{c}$ ), and resummation of diagrams with additional gluon radiation. In addition, the underestimation of the rates for diagrams with $g \rightarrow c \bar{c}$ splittings may result in lower theoretical predictions of cross sections. The prediction from the $k_{\mathrm{T}}$-factorization approach and Sherpa are in better agreement with data .

The D0 experiment has also measured the ratio of differential cross sections for $\gamma+c$-jet to $\gamma+b$-jet production in bins of $p_{T}^{\gamma}$. In this ratio, many experimental systematic uncertainties cancel. Also, theory predictions of the ratio are less sensitive to the effects from missing higherorder terms that impact the normalizations of the cross sections. Fig.4 shows the data to theory comparison for the ratio. There is good agreement with NLO, SHERPA and PYTHIA predictions in the region $30<p_{T}^{\gamma} \lesssim 70 \mathrm{GeV}$, while, at higher $p_{T}^{\gamma}$, data show systematically higher ratios where $k_{\mathrm{T}}$-factorization starts agreeing with data within uncertainties. The results are also compared with predictions with two intrinsic charm models. The BHPS model [12] agrees with data at larger $p_{T}^{\gamma}$.
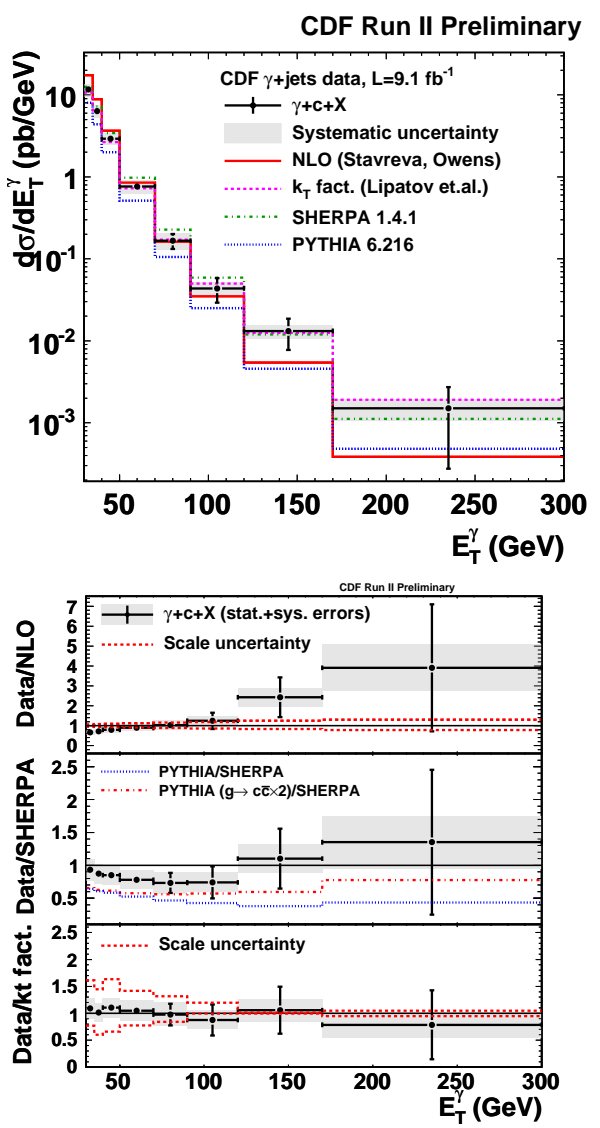

Figure 5. The measured differential cross sections compared with four theoretical predictions. The absolute comparisons are shown in the left plot and the ratios of data over the theory are shown in the right plot. The shaded area around the data points indicates total systematic uncertainty of the measurement.

\section{Inclusive diphoton production cross section}

Direct photon pair production constitutes a large and irreducible background in the study of Higgs boson decaying into a pair of photons and in searches for new phenomena, such as new heavy resonances, extra spatial dimensions or cascade decays of heavy new particles. In addition, diphoton production provides important check of the validity of pQCD predictions and soft-gluon resummation methods implemented in theoretical calculations.
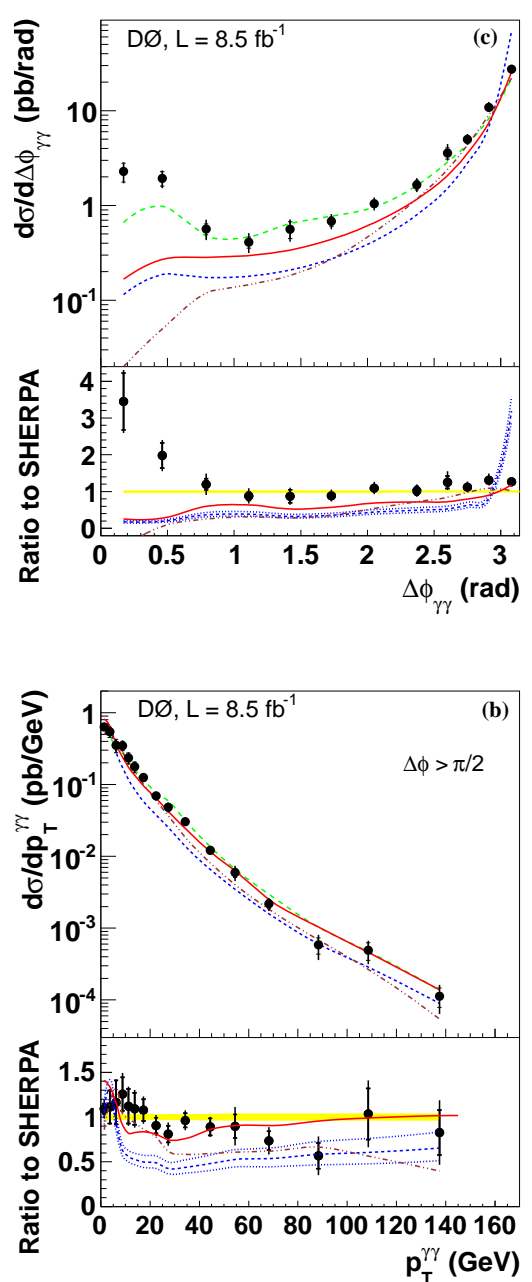

Figure 6. The differential diphoton cross section as a function of invariant diphoton mass , $M_{\gamma \gamma}$ (Upper) and transverse momentum of the diphoton system $p_{T}^{\gamma \gamma}$ (Lower) along with the predictions from DIPHOX, RESBOS , $2 \gamma \mathrm{NNLO}$, and SHERPA.

The D0 experiment has performed the measurements of of photon pair production cross sections using 8.5 $\mathrm{fb}^{-1}$ of data [13]. The results are presented as differential distributions of photon pair invariant mass $\mathrm{d} \sigma / \mathrm{d} M_{\gamma \gamma}$, pair transverse momentum $\mathrm{d} \sigma / \mathrm{d} p_{T}^{\gamma \gamma}$, azimuthal angle between the photons $\mathrm{d} \sigma / \mathrm{d} \Delta \phi^{\gamma \gamma}$, and polar scattering angle in the Collins-Soper frame $\mathrm{d} \sigma / \mathrm{d}\left|\cos \theta^{*}\right|$. Measurements are performed for isolated photons with transverse momenta $p_{T}^{\gamma}>18$ (17) $\mathrm{GeV}$ for the leading (next-leading) photon in $p_{T}^{\gamma},\left|y^{\gamma}\right|<0.9$, and a separation in $\eta-\phi$ space 
$\Delta R_{\gamma \gamma}>0.4$. The analysis splits the data into two subsets, $\Delta \phi_{\gamma \gamma} \geq \pi / 2$ and $\Delta \phi_{\gamma \gamma}<\pi / 2$ to isolate regions with smaller and larger relative contributions from the fragmentation process. The measurements are compared with the predictions DIPHOX, RESBOS , $2 \gamma$ NNLO , and SHERPA. Fig. 6 shows the comparison of the measured spectra as a function of $\Delta \phi^{\gamma \gamma}$ and $p_{T}^{\gamma \gamma}$ with the predictions. Overall, SHERPA has been found to provide the best agreement of the measurement cross sections. The results show discrepancies with all theoretical predictions in the regions of small $\Delta \phi_{\gamma \gamma}$ and small diphoton mass for $\Delta \phi_{\gamma \gamma} \geq \pi / 2$ with minor differences in the shapes of the $\left|\cos \theta^{*}\right|$ distribution.
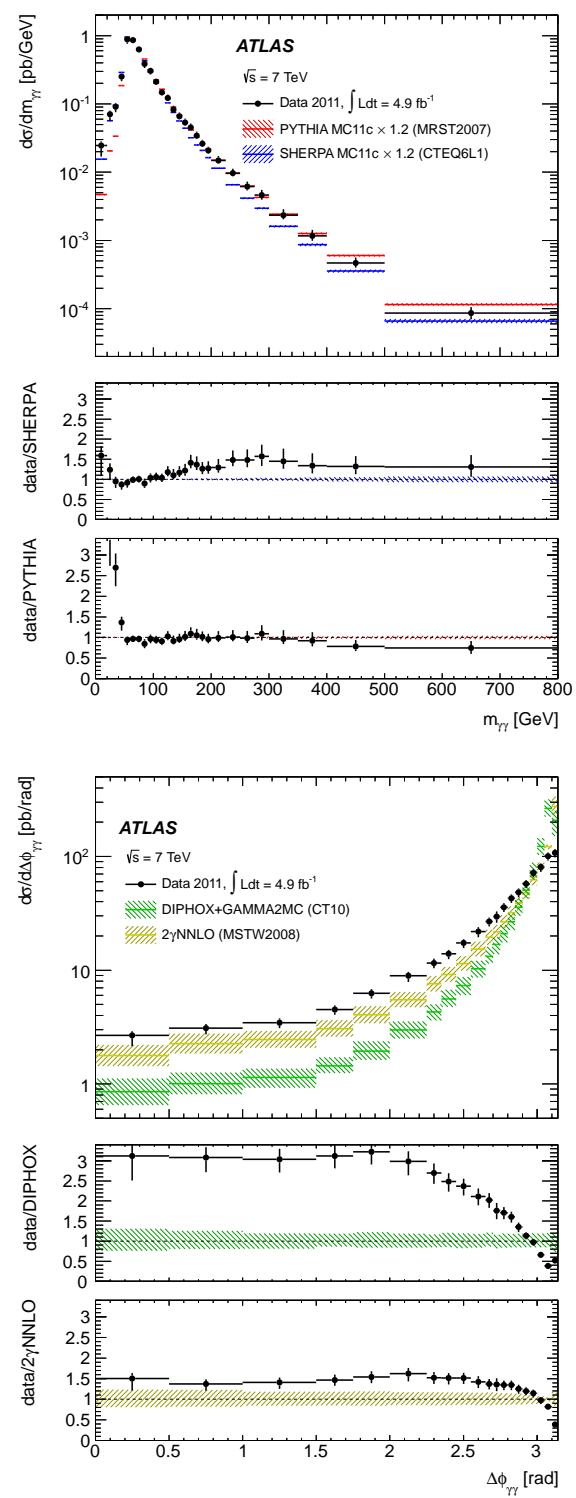

Figure 7. The differential diphoton cross section as a function of invariant diphoton mass $M_{\gamma \gamma}$ (Upper) along with the predictions from PYTHIA and SHERPA. The differential diphoton cross section as a function of difference in azimuthal angle between two photons $\Delta \phi_{\gamma \gamma}$ (Lower) along with the predictions from DIPHOX+Gamma2MC and $2 \gamma$ NNLO.
The ATLAS experiment has also measured these differential cross sections in $p p$ collisons at $\sqrt{s}=7 \mathrm{TeV}$ based on about $5 \mathrm{fb}^{-1}$ of data [14]. The measurement requires two isolated photons with $E_{T}^{\gamma}>25$ (22) $\mathrm{GeV}$, in the acceptance of the electromagnetic calorimeter ( $\left|\eta^{\gamma}\right|<1.37$ and $\left.1.52<\left|\eta^{\gamma}\right|<2.37\right)$ and with an angular separation $\Delta R_{\gamma \gamma}>0.4$. Rather good agreement is found with Monte Carlo generators, after rescaling the PYTHIA and SHERPA distributions by a factor of 1.2 in order to match the integrated cross section measured in data and the fixed order calculations (see Fig. 7). All generators tend to underestimate the data at large $\left|\cos \theta^{*}\right|$. SHERPA performs rather well for most differential spectra except for high $m_{\gamma \gamma}$. DIPHOX+Gamma2MC fails to match data at low $p_{T}^{\gamma \gamma}$ and $\Delta \phi_{\gamma \gamma} \sim \pi$ since it lacks resummation of soft-gluon emissions. Everywhere else DIPHOX+Gamma2MC is missing NNLO contributions and clearly underestimates the data (see Fig. 8). With the inclusion of NNLO terms, $2 \gamma \mathrm{NNLO}$ is able to match data very closely within uncertainties, except in limited regions where the fragmentation component - neglected in $2 \gamma$ NNLO calculation - is still significant.

\section{References}

[1] J.F. Owens, Rev. Mod. Phys. 59465 (1987).

[2] ATLAS Collaboration, Phys. Lett. B706 150 (2011).

[3] ATLAS Collaboration, Phys. Rev. D83 052005 (2011).

[4] P. Aurenche et. al., Phys. Rev. D73 094007 (2006).

[5] ATLAS Collaboration, Phys. Rev. D85 092014 (2012).

[6] T. Stavrera and J. Owens, Phys. Rev. D79 054017 (2009).

[7] V.M. Abazov et al. (DØ collaboration), Phys. Lett. B714 32 (2012).

[8] V.M. Abazov et al. (DØ collaboration), Phys. Lett. B719 354 (2013).

[9] A.V. Lipatov and N.P. Zotov, J. Phys. G 34, 219 (2007); S.P. Baranov, A.V. Lipatov, and N.P. Zotov, Eur. Phys. J. C 56, 371 (2008).

[10] T. Gleisberg et al., J. High Energy Phys. 02, 007 (2009).

[11] CDF CDF/PHYS/JET/PUBLIC/10818.

Collaboration,

[12] S.J. Brodsky, P. Hoyer, C. Peterson, N. Sakai, Phys. Lett. B 93, 451 (1980).

[13] V.M. Abazov et al. (DØ collaboration), arXiv:hepex/1301.4536.

[14] ATLAS Collaboration, JHEP 01086 (2013). 